\title{
Distinguishing black holes through their ringing
}

\author{
Shannon Potter, Luis Lehner*
}

\author{
*This study was conducted under the supervision of Professor Lehner, \\ Department of Physics, College of Physical and Engineering Science, \\ University of Guelph, Guelph, Ontario, Canada and \\ the Perimeter Institute for Theoretical Physics
}

\begin{abstract}
A perturbed black hole spacetime emits gravitational waves possessing quasinormal modes that are characteristic of the black hole itself. We use a massless scalar field as an analog to a gravitational wave to find the quasinormal modes emitted by both a Schwarzschild black hole and a new alternative black hole model which places the Schwarzschild black hole in an aether - a zero density, negative pressure perfect fluid. The later model was proposed as an alternative explanation for accelerated cosmic expansion [1]. We construct a computational code to study both systems numerically and obtain the corresponding quasinormal modes. We find that the quasinormal modes of a black hole in an aether are distinguishable from those of a Schwarzschild black hole and so, in principle, gravitational wave observations could be exploited to determine if either black hole solution represents those existing in our universe.
\end{abstract}

$\mathrm{W}^{\mathrm{h}}$ hen a black hole is disturbed in a certain way, for example during its formation from stellar collapse or during matter accretion, it will produce gravitational waves. These waves exhibit characteristic dampened oscillations, or quasinormal modes (QNMs) which are qualitatively analogous to those of a ringing bell. With the construction of gravitational wave detectors (such as LIGO in Livingston, LA and Hanford, WA and VIRGO in Italy which are operational, as well as LISA which is planned to orbit the Earth in 2018) these QNMs are expected to be observed, thereby opening a new window from which to scrutinize our universe. Key characteristics of QNMs, namely their frequency and decay, are independent of the initial disturbance to the black hole and depend only on the properties of the black hole[2]. For this reason, observed QNMs, when compared with theoretical QNMs, may serve as both a probe to the properties of a black hole and a means of testing gravitational theories. In this paper we study the theoretical QNMs produced by two different models. The first considers a black hole in a vacuum (the Schwarzschild solution), and the second in a fluid background proposed by Prescod-Weinstein et al [1], which arises in the context of theories attempting to accommodate for dark energy. The QNMs of the first model have been widely studied, the latter have not. Here we provide a first study with the goal of understanding whether gravitational waves can help to distinguish between these two possibilities.

\section{Metrics}

In the framework of Einstein's theory of General Relativity spacetime is curved by matter and energy, as described by the Einstein field equations:

$$
\frac{8 \pi G}{c^{4}} T_{\alpha \beta}=R_{\alpha \beta}-\frac{1}{2} g_{\alpha \beta} R+\Lambda g_{\alpha \beta}
$$

In equation (1) and what follows, Einstein summation convention is employed and there is a summation over repeated Greek indices. We use geometric units, whereby both the speed of light $(c)$ and the gravitational constant $(G)$ are unity. As a result, time has units of length, equal to the distance travelled by light during that time interval: $1 \mathrm{~s}=$ $3.0 \times 10^{8} \mathrm{~m}$. It follows that mass also has units of length since in SI units $G \propto \frac{\mathrm{m}^{3}}{\mathrm{kgs}^{2}}=1$, and relating seconds to metres gives a conversion from kilograms to metres. In (1) $R_{\alpha \beta}$ is the contraction of the Riemann curvature tensor (which encodes the curvature of spacetime), and $R$ is the trace of $R_{\alpha \beta}$. The metric tensor, $g_{\alpha \beta}$, provides the relative separation $(d s)$ of two neighbouring points in spacetime:

$$
d s^{2}=g_{\alpha \beta} \mathrm{d} x^{\alpha} \mathrm{d} x^{\beta}
$$

The source of curvature in (1) is the stress-energy tensor $T_{\alpha \beta}$ and $\Lambda$ is the cosmological constant proposed by Einstein in order to yield a stationary universe. The inclusion of non-zero $\Lambda$ leads to a residual $\left(T_{\alpha \beta}=0\right)$ curvature which has recently been exploited as a possible way to account for the accelerated expansion of the universe, although this is not without controversy [3]. In this paper we consider $\Lambda=$ 0 and the only source of the curvature is the stress-energy tensor, which is used in two forms: the first represents a vacuum $\left(T_{\alpha \beta}=0\right)$ and the second a zero-density, negative pressure perfect fluid.

The Schwarzschild solution [4] is the unique static, spherically symmetric solution to equation (1) which applies to both a black hole in a vacuum and the vacuum exterior of a star. The metric which characterizes the Schwarzschild 
black hole, where $M$ is the mass of the black hole in meters and $G=c=1, \quad$ is given by [4]: $d s^{2}=-\left(1-\frac{2 M}{r}\right) d t^{2}+\left(1-\frac{2 M}{r}\right)^{-1} d r^{2}+r^{2} d \Omega^{2}$

The second metric examined [1] places the Schwarzschild black hole in an 'aether' - a negative pressure, zero-density perfect fluid - the metric is obtainable from hydrstatic equilibrium conditions [5]. The appeal of this new metric is that instead of attributing the accelerated expansion to a non-zero $\Lambda$ in (1), the acceleration arises naturally as a result of the presence stellar mass black holes in an aether background, as shown by Prescod-Weinstein et al [1]. The metric is [1]:

$$
\begin{gathered}
d s^{2}=-e^{2 \phi} d t^{2}+\left(1-\frac{2 M}{r}\right)^{-1} d r^{2}+r^{2} d \Omega^{2} \\
e^{\phi}=\left(1-\frac{2 M}{r}\right)^{1 / 2}\left[4 \pi p_{0} f(r)+1\right] \\
f(r)=\frac{1}{2}\left(1-\frac{2 M}{r}\right)^{-\frac{1}{2}}\left(-30 M^{2}+5 M r+r^{2}\right) \\
+\frac{15}{2} M^{2} \ln \left[\frac{r}{M}-1\right. \\
\left.+\frac{r}{M}\left(1-\frac{2 M}{r}\right)^{-\frac{1}{2}}\right]
\end{gathered}
$$

The aether pressure is given by $p=p_{0} e^{-\phi}$, and its value is fixed by requiring that the metric account for the accelerated expansion in the same way as the concordance model. That is, it is matched to a de Sitter metric far from the black hole [1].

Both black holes studied are non-charged and nonspinning. The properties of the aether black hole spacetime are therefore limited to fluid pressure and black hole mass, whereas Schwarzschild black holes can only vary in mass. For the purposes of simulating these black hole spacetimes, the mass solely determines the location of the event horizon and as the perturbations considered do not significantly affect black hole mass, we keep it constant.

\section{Quasinormal modes}

Any energy distribution with a non-zero time varying quadrupole (or higher multipole) moment produces gravitational waves (GWs) on the curved spacetime background. Both the Schwarzschild and the aether metrics describe static, spherically symmetric black holes. Gravitational, electromagnetic, or scalar waves scatter in these spacetimes and probe the behavior of generic perturbations. The waves scattered exhibit quasinormal modes (QNMs) which have been studied extensively since they were first introduced in 1970 [2]. It was shown then that the main characteristics of the QNMs are independent of the initial (gravitational or otherwise) perturbation and dependent only on the properties of the black hole.

GWs interact with the background curvature and the equations modelling their propagation are complex. It is simpler to model GWs using a scalar (instead of gravitational) perturbation, as this captures the general behaviour without gauge issues obscuring the analysis. For this reason we use a massless scalar field analog to find the QNMs of both black hole models considered. With the current development of gravitational wave detectors, our results are useful as they show that an aether background is distinguishable (at least in principle) in the QMN spectrum from a vacuum background, and the relationship found is expected to carry over to the realistic case of gravitational perturbations.

\section{METHODS}

The equation that describes the scalar field in curved spacetime is the Klein-Gordon Equation, which is a relativistic generalization of the wave equation:

$$
\left(\frac{\partial^{2}}{\partial t^{2}}-\nabla^{2}\right) \Phi=0 \rightarrow \frac{1}{\sqrt{-g}} \frac{\partial}{\partial x^{\mu}}\left(\sqrt{-g} g^{\mu \nu} \frac{\partial \Phi}{\partial x^{\mu}}\right)=0
$$

In (4) $g$ is the determinant of $g_{\alpha \beta}$ and $g^{\alpha \beta}$ is the inverse of $g_{\alpha \beta}$. The metrics in (2) and (3) are singular at the event horizon and so before applying (4) to (2), the coordinates are changed according to the Eddington-Finkelstein coordinate transformation $[6,7]$ :

$$
\begin{gathered}
t^{\prime}=t-r^{*}+r \\
r^{*}=r+2 M \ln \left|\frac{r}{2 M}-1\right|
\end{gathered}
$$

This transformation removes the coordinate singularity in the Schwarzschild metric but it does not remove the singularity in the aether metric so in this case we use an inner boundary $r_{0}>2 M$. For the aether metric the constant $p_{0}$ is given in terms of the trans-Plankian parameter $\theta_{p}$, as specified by Prescod-Weinstein et al.: $p_{0}=-\frac{1}{256 \pi^{2} \theta_{p} M^{3}}$. The pressures we look at correspond to $\theta_{p}=30-500$, which is the range suggested to be realistic by PrescodWeinstein et al. We find that in addition to the event horizon at $r=2 M$, coordinate singularities arise for high pressures $\left(\theta_{p}<120\right)$ at $r_{0} \cong 2.02 M$. We have compared the results obtained for choices of $r_{0}=2.03 M \ldots 2.11 M$ for the extreme cases of high pressure: $l=1, l=4$, with $\theta_{p}=30$ and $\theta_{p}=120$. We found that the results are independent of $r_{0}$, for small $r_{0}$, and we have used $r_{0}=2.05$ for $\theta_{p}<120$. For $\theta_{p} \geq 120$, the only coordinate singularity is at $r=2 M$ so it is possible to begin just outside the horizon $\left(r_{0} \cong 2.001 M\right)$. Because we are looking for the relationship between the frequency, decay and $\theta_{p}$ for the entire range $30 \leq \theta_{p} \leq 500$ we verify that solutions for $\theta_{p} \geq 120$ and the Schwarzschild case are not altered when using $r_{0}=2.05$. This allows us to freely compare the results within $30 \leq \theta_{p} \leq 500$ and the Schwarzschild case, $p_{0}=0$.

For both metrics, the solution $\Phi$ is decomposed as: $\Phi=\sum_{l} \chi(r, t) Y_{m}^{l}(\theta, \phi)$ where $Y_{m}^{l}(\theta, \phi)$ is a spherical harmonic, and solutions are found for a given value of $l$ (since, as both metrics are spherically symmetric, $m$ can be set to zero without loss of generality). 
There is no closed form analytic solution to the partial differential equation (4) when applied to the metrics. For this reason numerical integration is used to find the solution $\Phi(\mathrm{r}, \mathrm{t})$ from an initial wave $\Phi(\mathrm{r}, \mathrm{t}=0)$, and its time derivative $\pi=\frac{\partial}{\partial t} \Phi(\mathrm{r}, \mathrm{t}=0)$.

The initial wave is a pulse centered at $r=10 M$ (just outside the horizon at $r=2 M$ ) which serves as a perturbation to the black hole. Since the features we are concerned with in the QNMs are independent of the form of the perturbation the only requirement is that the pulse be smooth. The initial radial wave used is $\Phi(r, t=0)=$ $e^{-(r-10 M)^{2}}$, although simulations were done with smooth variations of $\Phi(r, t=0)$ as a means to verify that the QNMs found are in fact independent of the initial perturbation.

The integration is done using a code written in Matlab. The second order partial differential equation of (4) is written as two differential equations which are first order in time:

$$
\begin{gathered}
\pi=\frac{\partial \Phi}{\partial \mathrm{t}} \\
\frac{\partial \pi}{\partial t}=-f_{1}(r) \frac{\partial^{2} \Phi}{\partial \mathrm{r}^{2}}-f_{2}(r) \frac{\partial \pi}{\partial \mathrm{r}}-f_{3}(r) \frac{\partial \Phi}{\partial \mathrm{r}}-f_{4}(r) \pi \\
-f_{5}(r) \mathrm{l}(\mathrm{l}+1) \Phi
\end{gathered}
$$

This approach allows us to employ a set of robust numerical techniques that guarantee stability of the resulting implementation. The evolution of $\Phi(r, t)$ (the numerical solution to (4)) is found using the Runge-Kutta third order method.

For the Schwarzschild solution the functions $f_{n}(r)$ are dependent on the radial distance $r$ and the mass of the black hole $M$, and for the aether solution $f_{n}(r)$ are dependent on $r$, $M$, and the aether pressure $p$. To employ the Runge-Kutta method the equations (6) and (7) must be written discretely, using finite difference equations to represent the partial derivatives. Representing the discrete formulation of (7) as $L\left(\pi^{n}, \Phi^{n}\right)$, the values of $\Phi^{n+1}$ and $\pi^{n+1}$ (the solutions at time $\Delta t$ later than $\Phi^{n}$ and $\pi^{n}$ ) are found from $\Phi^{n}$ and $\pi^{n}$ in three steps. First $\Phi$ and $\pi$ are found at the midpoint, $\frac{1}{2} \Delta t$ later:

$$
\begin{gathered}
\pi^{(1)}=\pi^{n}+\frac{1}{2} \Delta t \cdot L\left(\pi^{n}, \Phi^{n}\right) \\
\Phi^{(1)}=\Phi^{n}+\frac{1}{2} \Delta t \cdot \pi^{(1)}
\end{gathered}
$$

Second the solutions are found after a time $\frac{3}{4} \Delta t$, using the results from the previous step.

$$
\begin{gathered}
\pi^{(2)}=\pi^{n}+\frac{3}{4} \Delta t \cdot L\left(\pi^{(1)}, \Phi^{(1)}\right) \\
\Phi^{(2)}=\Phi^{n}+\frac{3}{4} \Delta t \cdot \pi^{(2)}
\end{gathered}
$$

Finally the solutions a full $\Delta t$ later are found using the intermediate $\pi, \Phi$ :

$$
\begin{gathered}
\pi^{\mathrm{n}+1}=\pi^{n}+\frac{1}{9} \Delta t \cdot\left(2 L\left(\pi^{n}, \Phi^{n}\right)+3 L\left(\pi^{(1)}, \Phi^{(1)}\right)+4 L\left(\pi^{(2)}, \Phi^{(2)}\right)\right) \\
\Phi^{\mathrm{n}+1}=\Phi^{n}+\frac{1}{9} \Delta t \cdot\left(2 \pi^{\mathrm{n}}+3 \pi^{(1)}+4 \pi^{(2)}\right)
\end{gathered}
$$

After the radial solution is found at each intermediate time step, a high pass filter is applied to prevent long term instabilities. This dissipation modifies the solutions $(\pi, \Phi)$ by an amount $\epsilon<0$ of the discrete fourth partial derivative with respect to radial distance $(\pi$, rrrr,$\Phi$,rrrr $)$ as shown in (11)-(13) where $F=\{\pi, \Phi\}$. This modification is done before proceeding to the next step in the integration (the equations are employed in the sequence (8), (11), (9), (12), (10), (13)).

$$
\begin{aligned}
& \mathrm{F}^{(1)}=\mathrm{F}^{(1)}+\frac{1}{2} \epsilon \Delta t \mathrm{~F}_{\text {,rrrr }}^{(1)} \Delta r^{3} \\
& \mathrm{~F}^{(2)}=\mathrm{F}^{(2)}+\frac{3}{4} \epsilon \Delta t \mathrm{~F}_{\text {,rrrr }}^{(2)} \Delta r^{3} \\
& \mathrm{~F}^{\mathrm{n}+1}=\mathrm{F}^{n+1}+\frac{1}{9} \epsilon \Delta t\left(2 \mathrm{~F}_{\text {,rrrr }}^{\mathrm{n}}+3 \mathrm{~F}_{\text {,rrrr }}^{(1)}+4 \mathrm{~F}_{\text {,rrrr }}^{(2)}\right) \Delta r^{3}
\end{aligned}
$$

Clearly, in the limit $\Delta t \rightarrow 0, \Delta r \rightarrow 0$ the above dissipation does not alter the solution; however, outside of it, the dissipation helps to control the highest frequency modes which are never represented faithfully by discrete approximations and, typically drive instabilities. The introduction of this dissipation ensures stability without negatively affecting the convergence of the implementation.

The discrete representations of the derivatives in (7) used in $L\left(\pi^{n}, \Phi^{n}\right)$ are given in (14). The indexing $i$ labels the discrete radial values, quantized by $\Delta r$.

$$
\begin{aligned}
\left.\frac{\partial^{2} \Phi}{\partial \mathrm{r}^{2}}\right|_{i} & \rightarrow \frac{\Phi^{\mathrm{n}_{\mathrm{i}+1}-2 \Phi^{\mathrm{n}}{ }_{\mathrm{i}}+\Phi^{\mathrm{n}} \mathrm{i}-1}}{\Delta \mathrm{r}^{2}} \\
\left.\frac{\partial \pi}{\partial \mathrm{r}}\right|_{i} & \rightarrow \frac{\pi^{\mathrm{n}}{ }_{\mathrm{i}+1}-\pi^{\mathrm{n}}{ }_{\mathrm{i}-1}}{2 \Delta \mathrm{r}} \\
\left.\frac{\partial \Phi}{\partial \mathrm{r}}\right|_{i} & \rightarrow \frac{\Phi^{\mathrm{n}}{ }_{\mathrm{i}+1}-\Phi^{\mathrm{n}}{ }_{\mathrm{i}-1}}{2 \Delta \mathrm{r}}
\end{aligned}
$$

These discretizations follow from finding the sum and difference of the Taylor expansions of $\Phi(\mathrm{r}+\Delta \mathrm{r}), \Phi(\mathrm{r}-\Delta \mathrm{r})$, and likewise with $\pi$. The fourth partial derivatives used in the dissipation are discretized as:

$$
\mathrm{F}_{\text {,rrrr }}^{\mathrm{n}}=\frac{\mathrm{F}^{\mathrm{n}}{ }_{\mathrm{i}+2}-4 \mathrm{~F}_{\mathrm{i}+1}^{\mathrm{n}_{1}}+6 \mathrm{~F}_{\mathrm{i}}^{\mathrm{n}}-4 \mathrm{~F}_{\mathrm{i}-1}^{\mathrm{n}_{1}}+\mathrm{F}_{\mathrm{i}+2}^{\mathrm{n}}}{\Delta \mathrm{r}^{4}}
$$

Variable integration steps are used with the restriction $\Delta t=\lambda \Delta \mathrm{r}, \lambda<1$ in compliance with the CFL condition [8] for numerical stability: at a time $t^{n}$, the value of the solution $\Phi\left(r, t^{n}\right)$ can only depend on a certain subdomain of the field a time $\Delta t$ earlier. This is known as the domain of dependence of $\Phi$ and is defined as the region of points at $t^{n-1}$ which can determine the value of $\Phi\left(r, t^{n}\right)$. Since signals can propagate at most at the speed of light $c$, the CFL condition restricts $\frac{\Delta t}{\Delta r} \leq c$ (in the units we use, $c=1$ ). For both the Schwarzschild and aether solutions we use $\lambda=0.5$ to ensure this condition is satisfied.

QNMs are exhibited by the field over time, and the time derivative of the field is fit to a a function of the form $y=e^{\alpha t} \sin (\omega t)$, where $\alpha$ is the decay and $\omega$ is the frequency of the QNM. As we perform a numerical integration, in addition to physical parameters, the decays and frequencies we obtain are dependent on the size of the integration step $\Delta r$, as well as the location of the observer: the radial distance from the black hole, $r$, at which the QNM is fit. These dependencies can be accounted for as discussed 


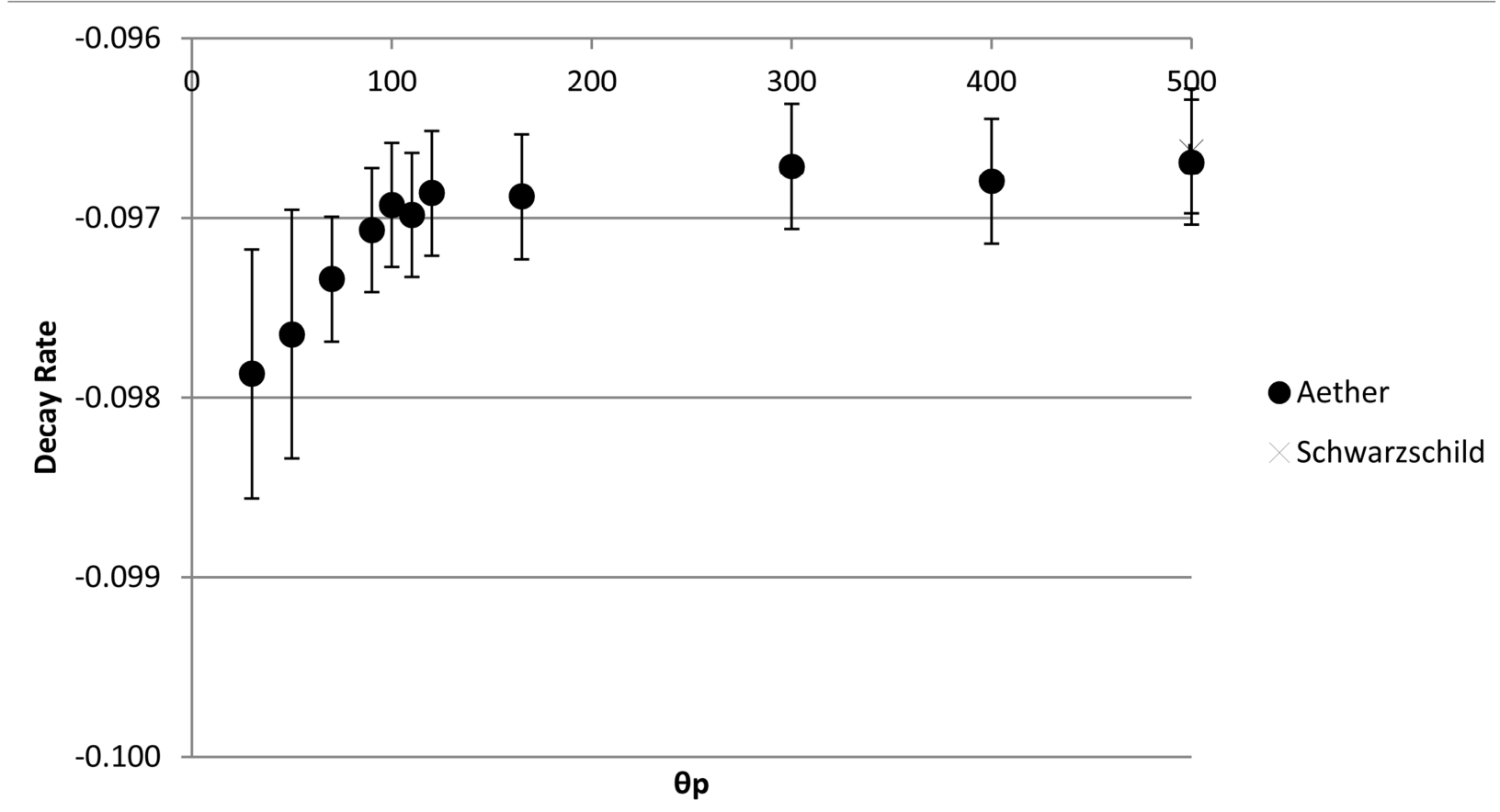

Figure 1: Decay of $\mathrm{l}=3$ modes for different values of $\boldsymbol{\theta p}$.

below and their spurious influence removed from the predicted values.

It is known that the true value, $\alpha(\omega)$, is related to the value observed at some location $r, \alpha_{o b s}\left(\omega_{o b s}\right)$, by:

$$
\alpha_{o b s}=\alpha+\frac{c}{r}
$$

where $\mathrm{c}$ is a constant. To find $\alpha$ (or identically, $\omega$ ), a set of observations are made and a linear fit is done to $r \alpha_{o b s}$ as a function of $r$ in order to yield the value of $\alpha$ as the slope:

$$
r \alpha_{o b s}=r \alpha+c
$$

The numerical solution approaches the continuous solution as $\Delta r \rightarrow 0$, and the true values of $\alpha, \omega$ are defined by the y-intercept of a curve fitting of $\alpha(\Delta r), \omega(\Delta r)$. The parameters $\alpha, \omega$ are found for several values of $\Delta r$ (with $\Delta t=0.5 \Delta r)$ for the extreme cases of $l=1, l=4$, with $\theta_{p}=30$ and $\theta_{p}=120$, and $p_{0}=0$. It is found that these solutions converge for $\Delta r \leq 0.033$ at which point the results $(\alpha, \omega)$ become independent of $\Delta r, \Delta t$ within uncertainty. For this reason an integration step of $\Delta r=0.033$ is sufficient to find the true solution and this is the value we use for all $l, \theta_{p}$.

\section{RESULTS}

The decay rate of the QNMs for $l=3$ and $l=4$ are shown in Figures 1 and 2. Although solutions were found for $l=0, l=1$ and $l=2$, no relationship was observed between decay rate of these modes and the aether pressure. Likewise, the frequencies were found for all modes but no changes were observed with increasing pressure. It may be that the pressure does not affect the frequency (and the decay of lower modes), or, more likely, that we were unable to observe the difference at our precision. For $l=3$ and $l=4$ a decrease in $\theta_{p}$ (an increase in pressure) corresponds to an increase in the decay rate as $\theta_{p}$ decreases below 120 .

The curve fitting of $\frac{\partial \Phi}{\partial \mathrm{t}}$ to the form $y=e^{\alpha t} \sin (\omega t)$ is done automatically first, to obtain an estimate of the values of $\alpha$ and $\omega$. To increase precision, the values of $\alpha, \omega$ are then manually adjusted and the goodness of fit visually observed. By this method, a small range of values may fit such that no adjustments (increase or decrease of $\alpha$ or $\omega$ ) appear to be necessary. The uncertainties, $\sigma_{\alpha}$ and $\sigma_{\omega}$, assigned to each value of $\alpha, \omega$ account for this range. As $\alpha, \omega$ are taken from different locations $r$ and the true values are obtained by weighted linear regression (weighed by $\sigma_{\alpha}$ and $\sigma_{\omega}$ ), the error bars shown in Figures 1-2 represent the uncertainty from the linear regression.

Results are shown for pressures corresponding to $\theta_{p}=30-500$ as well as the Schwarzschild case. We verified that the Schwarzschild decay rates $(\alpha)$ and frequencies $(\omega)$ are equal to the values at $p_{0}=0$, or $\theta_{p} \rightarrow \infty$. The frequencies used for comparison with the Schwarzschild case are those found using an analytic approach (a modification of the WKB method) and given by Iyer [9].

\section{DISCUSSION}

The results for $\alpha$ and $\omega$ in the Schwarzschild case are equal (within uncertainty) to the WKB values. In the cases $l=0$ to $l=2$, our results suggest that the presence of an aether is undetectable in both the decay rate and the frequency of the QNMs for all pressures. The trend is different in the $l=3$ 


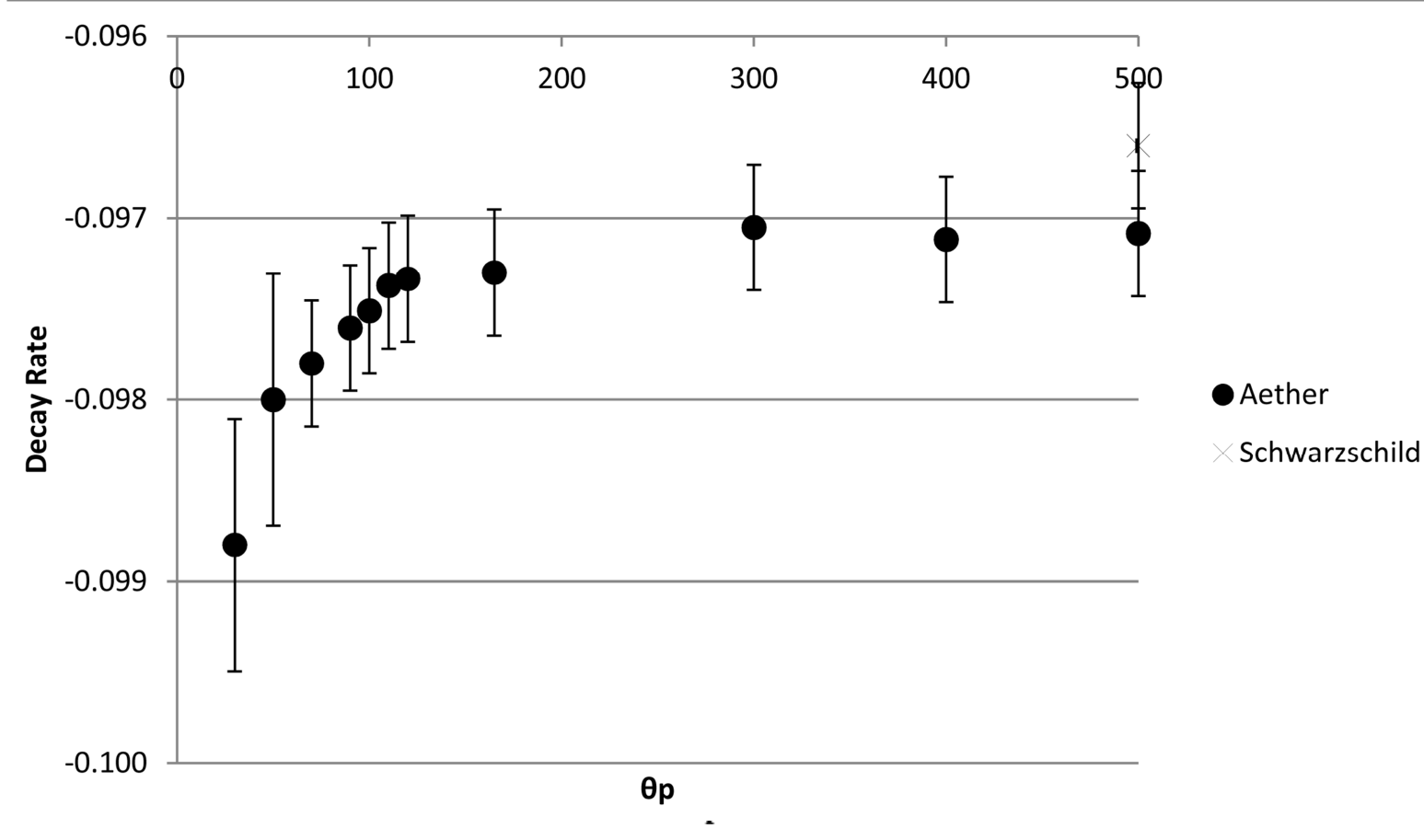

Figure 2: Decay of $\mathrm{l}=4$ modes for different values of $\theta p$.

and $l=4$ cases and the decay rate increases as the aether pressure increases, however thre is no pressure dependence observed in the frequency. It may be that there is no dependence, or alternately we were unable to observe it.

Any observed gravitational wave will posses all of the $l>0$ modes. Our results show that, in conjunction with one another, the $l$ modes of QNMs can in principle be used to distinguish a high pressure aether background from a vaccum and furthermore determine the aether pressure. Our results are unable to distinguish lower pressures $\left(\theta_{p}>120\right)$ from the vacuum.

As the trends in the decay rate appear to be more pronounced in higher modes, in the future finding the QNMs for $l>4$, in addition to increasing our precision would improve our understanding of the role of QNMs in distinguishing these two backgrounds.

\section{CONCLUSION}

Quasinormal modes have been found for the Schwarzschild black hole and a black hole in an aether-a zero-density, negative pressure perfect fluid. It was verified that our Schwarzschild values agree with the analytical values and it was found that the presence of an aether modifies the decay rate of the QNM spectra from the Schwarzschild case.

The usefulness of our results is limited by having looked at a scalar and not gravitational perturbations: observable gravitational waves will possess QNMs which differ from those found using the scalar analog. As well they are expected to be produced by spinning black holes.
However QNMs from scalar perturbations of variations of non-charged, non-spinning black holes are widely studied in the literature and the QNMs found here are able to be compared with those arising in these other models. Furthermore, the qualitative relationship we have found is expected to carry over to realistic black holes: the presence of an aether will alter the QNMs of a black hole from those in a vacuum.

\section{ACKNOWLEDGEMENTS}

This research has been supported in part by NSERC through a Discovery Grant.

\section{REFERENCES}

1. Stellar black holes and the origin of cosmic acceleration. Prescod-Weinstein, C, Ashfordi, N and Balogh, M L. s.1. : Physical Review D, 2009, Vol. 80. 043513.

2. Stability of the shcwarzschild metric. Vishveshwara, C. s.l. : The American Physical Society, 1970.

3. The Cosmic Energy Inventory. Fukugita, Masataka and Peebles, P. J. E. s.l. : The Astrophysical Journal, 2004.

4. On the gravitation field of a mass point according to einstein's theory. Schwarzschild, K. 1999, The American Physical Society. Translated version, by S Antonci and A Loinger. 
5. On Massive Neutron Cores. Oppenheimer, J R and Volkoff, G M. California : Physical Review, 1939, Vol. 55.

6. Eddington, A S. s.1. : Nature, 1924.

7. Finkelstein, D. s.l. : Physical Review, 1968.

8. On the partial difference equations of mathematical physics. Courant, R, Friedrichs, K and Lewy, H. s.l. : IBM Journal, 1967, Vol. 100.
9. Black-hole normal modes: A wkb approach. ii. schwarzschild black holes. Iyer, S. 12, s.l. : Physical Review D, 1987, Vol. 35.

10. Quasi-Normal Modes of Stars and Black Holes. Kokkotas, Kostas D and Schmidt, Bernard. 2, s.l. : Living Reviews in Relativity, 1999, Vol. 2. 